\title{
DARHT II Scaled Accelerator Tests on the ETA II Accelerator ${ }^{*}$
}

J. T. Weir, E. M. Anaya Jr, G. J. Caporaso, F. W. Chambers, Y.-J. Chen, S. Falabella, B. S. Lee, A. C. Paul, B. A. Raymond, R. A. Richardson, J. A. Watson, D. Chan, H. A. Davis, L. A. Day, R. D. Scarpetti, M. E. Schultze, T. P. Hughes

May 27, 2005

IEEE Pulse Power Conference Monterey, CA, United States June 13, 2005 through June 17, 2005 
This document was prepared as an account of work sponsored by an agency of the United States Government. Neither the United States Government nor the University of California nor any of their employees, makes any warranty, express or implied, or assumes any legal liability or responsibility for the accuracy, completeness, or usefulness of any information, apparatus, product, or process disclosed, or represents that its use would not infringe privately owned rights. Reference herein to any specific commercial product, process, or service by trade name, trademark, manufacturer, or otherwise, does not necessarily constitute or imply its endorsement, recommendation, or favoring by the United States Government or the University of California. The views and opinions of authors expressed herein do not necessarily state or reflect those of the United States Government or the University of California, and shall not be used for advertising or product endorsement purposes. 


\title{
DARHT II Scaled Accelerator Tests on the ETA II Accelerator*
}

\author{
John T. Weir, Enrique M. Anaya Jr., George J. Caporaso, Frank W. Chambers, Yu-Jiuan \\ Chen, Steven Falabella, Bryan S. Lee, Arthur C. Paul, Brett A. Raymond, Roger A. \\ Richardson, and James A. Watson \\ Lawrence Livermore National Laboratory \\ Livermore, California*
}

\author{
Dominic Chan, Harold A. Davis, Lisa A. Day, and Raymond D. Scarpetti \\ Los Alamos National Laboratory \\ Los Alamos, New Mexico \\ Martin E. Schultze \\ General Atomics \\ Los Alamos, New Mexico \\ Thomas P. Hughes \\ ATK Mission Research Corp. \\ Albuquerque, New Mexico
}

\begin{abstract}
The DARHT II accelerator at LANL is preparing a series of preliminary tests at the reduced voltage of 7.8 $\mathrm{MeV}$. The transport hardware between the end of the accelerator and the final target magnet was shipped to LLNL and installed on ETA II. Using the ETA II beam at $5.2 \mathrm{MeV}$ we completed a set of experiments designed reduce start up time on the DARHT II experiments and run the equipment in a configuration adapted to the reduced energy. Results of the beam transport using a reduced energy beam, including the kicker and kicker pulser system will be presented.
\end{abstract}

\section{INTRODUCTION}

As a preliminary experiment in the commissioning of the DARHT II beamline, the down stream transport equipment including the Kicker and Septum were brought to LLNL and tested with the electron beam of the ETA II accelerator. The nominal parameters of ETA II are 5.2 MeV., $1.8 \mathrm{kA} .$. , and $40 \mathrm{~ns}$. pulse width. The accelerator runs at one pulse per second. The DARHT II hardware was designed for an $18 \mathrm{MeV}$. beam and in order to scale our experiment correctly the ETA II beam was reduced to 600 amps by using a graphite aperture plate after the end of the accelerator.

\footnotetext{
*This work was performed under the auspices of the U.S. Department of Energy, by the University of California, Lawrence Livermore National Laboratory, under Contract W-7405-Eng-48
}

In addition to this, we added one more solenoid (S4) to the transport line and increase the diameter of the beam pipe to accommodate a larger radius beam.

In the normal mode of operations, the Kicker Bias Dipole (a cosine wound coil around the Kicker) is active, and the beam is bent down as it enters the Septum Quadrupole (SQ). This amplifies the bend angle and the beam enters the Septum Dipole magnet and completes its 45 degree bend into the Septum Dump. When the Kicker is pulsed, it overcomes the effect of the Kicker Bias Dipole and the beam enters the Septum Quadrupole on axis and continues through the quadrupole region, transitions back to solenoid transport and continues to the final focusing magnet and the target.

A block diagram of the experimental configuration is shown in figure 1.

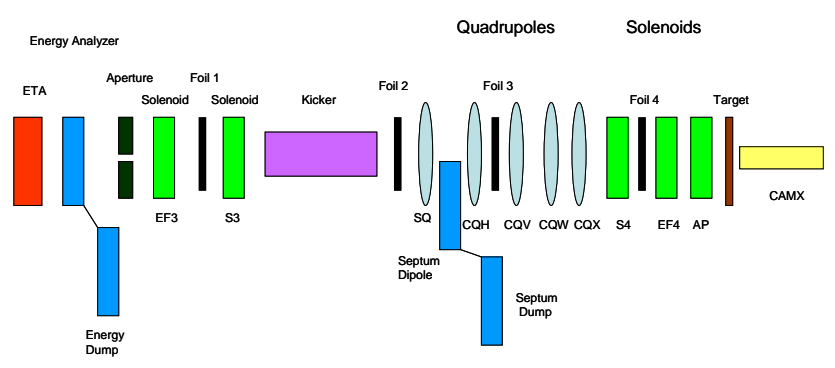

Figure 1.

By selecting the Kicker on times and durations we can choose the pulses we send to the target area. This is shown schematically in figure 2 . 


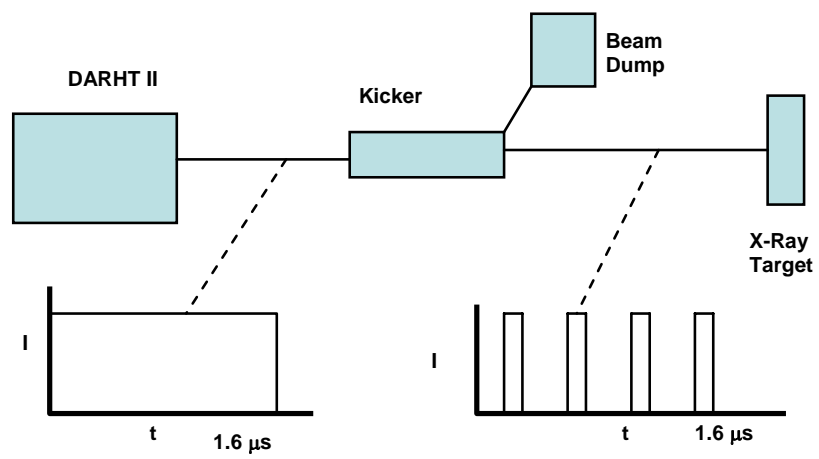

Figure 2.

\section{OBJECTIVES}

The primary objective was to develop a method for tuning the beam and transporting through the magnet system to the target while minimizing the number of shots to accomplish this. ETA II has the luxury of almost unlimited shots ( running at 1 pps.) and a short pulse length (40 ns.). We were able to view the beam using Cherenkov foils at any location we desired and tune the beam in a real time mode as we observed the foils with intensified cameras.

When we run the hardware at DARHT II, we will be limited to about 20 shots a day (average) and the long pulse ( $2 \mu \mathrm{sec}$.) makes it difficult to view the beam without destroying the foil. By running the preliminary tests on ETA II we were able ramp up the learning curve very quickly and debug systems without using the very valuable DARHT II shots to do this.

A secondary objective of the ETA II tests was to work closely with the DARHT II experimental team and to transfer the LLNL technology to LANL. This included all aspects of installation and alignment, operation of the Kicker and the Kicker pulsers, and comparing the LANL and LLNL beam codes against the experimental data. Once again, this was done to minimize the learning curve when the hardware is installed at DARHT II.

\section{RESULTS}

After running the ETA II beam through the graphite aperture, we needed to characterize the resultant 600 amp beam. This was done by observing the Cherenkov light produced by the beam hitting a thin quartz foil (foil 1) and measuring the beam radius. By varying a solenoid (EF3) up stream of the foil and measuring the beam radius we were able deduce the radius (R), the divergence ( $\mathrm{R}^{\prime}$ ), and the emittance (e) of the beam at a given point in the beam line (the aperture plate). The current (I) was measured with resistive wall
monitors(Beam Bugs) and the energy (E) was measured using the ETA II energy analyzer just upstream of the aperture plate. Typical values for these parameters were as follows:

$\mathrm{R}=0.67 \mathrm{~cm}$

$\mathrm{R}^{\prime}=11.1 \mathrm{mrad}$

$\mathrm{e}=42.7 \pi \mathrm{cm}-\mathrm{mrad}$ (normalized)

$\mathrm{I}=600 \mathrm{amps}$

$\mathrm{E}=5.25 \mathrm{MeV}$

Once the initial conditions of the beam had been measured we used the LLNL envelope code, FITS, to set the solenoids and quadrupoles used to transport the beam through the Kicker and Septum regions. This was done without energizing the Kicker (Kicker and Bias Dipole were both off). By concentrating on the magnetic tune and observing the beam on foils 2,3 and 4, we were able to optimize the transport through this section.

Once we had established a baseline magnetic tune we energized the Kicker Bias Dipole and then the Kicker Pulser. By observing the position of the beam on a Beam Position Monitor we were able to plot the Pulser voltage necessary to overcome different Bias Dipole settings. This data is shown in figure 3 .

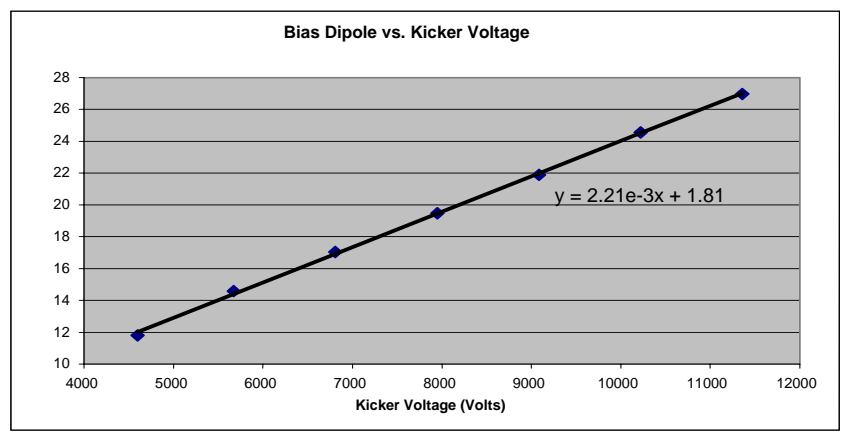

Figure 3.

Inherent in the operation of the Kicker is a sextupole error field produced by the activation of the Kicker. In order to compensate for this error field there is a sextupole winding around the exterior of the Kicker. For the parameters we ran on ETA II we found we needed 30 amps in the Sextupole corrector coil to buck out the error field.

With the Bias Dipole and Sextupole corrector set, we began using the Kicker to switch the beam from the Septum Dump to the straight ahead transport line. The Kicker has a feed forward control system that measures the deflection of the beam as a function of time through the pulse and does successive corrections on each shot until it has achieved the desired deflection. A plot of vertical displacement $(\mathrm{cm})$ vs. time is shown in figure 4 . 


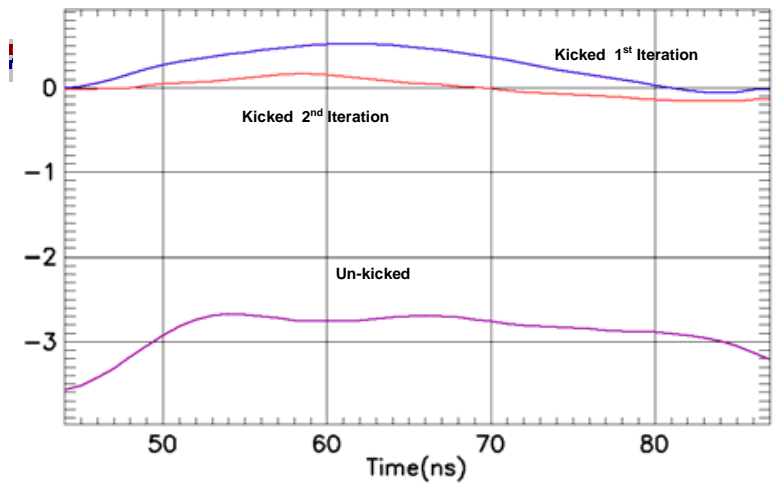

Figure 4.

Initial work on this system was done using the ETA II beam bugs and the ETA II data acquisition system. With the help the LANL team we transitioned the system to work with the LANL Beam Position Monitors (RF loops) and the LANL data acquisition system. The data shown in figure 4 was taken using the LANL system.

After running through the Kicker system and the quadrupole magnets the beam transitioned back to solenoid transport. Using these solenoids we brought the beam to the final focus magnet (AP) and focused it down on the target. Our targets were 0.003 inch thick Ta. foils. The X-rays produced by the beam/target interaction were observed with the time resolved X-ray camera (CAMX). Examination of the X-ray image of the kicked beam on target showed we were successful in running the entire hardware package and delivering a useful beam to the target. Figure 5 shows a comparison of the kicked vs. the un-kicked beam as seen by CAMX on the target. Each frame is a 10 ns snap shot of the beam with 10 ns steps between frames..

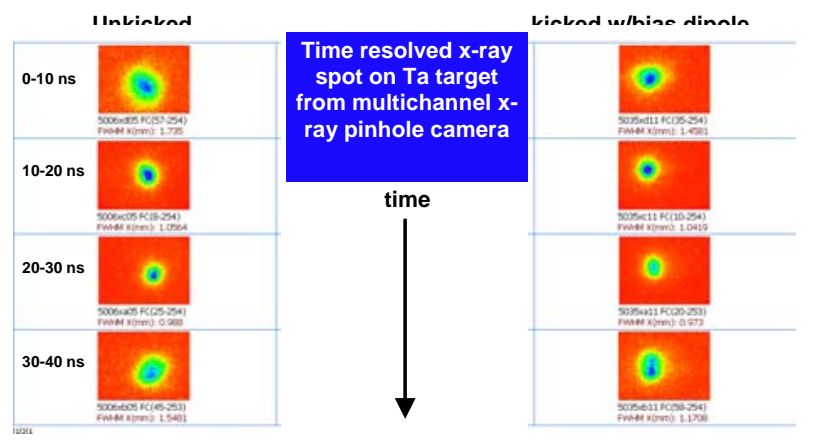

Figure 5.

Throughout the experiments listed above we depended on beam codes to predict settings of the magnets as we advanced the beam through the transport section. The two codes we used were LAMBDA and FITS. Comparisons between the two codes were run using identical input parameters and we had excellent agreement between the codes.

In order to compare the codes to experimental data we would typically start with a set of input parameters gathered by scanning a magnet in front of an observation foil and using the codes to back out the input conditions. We would then run the beam through the next magnet (or magnets) to another observation foil and compare the measured data to the predicted size (and shape if we ran through quadrupoles) we observed. Figure 6 shows the optical data we collected when scanning the CQX magnet and observing the beam at foil 4 .

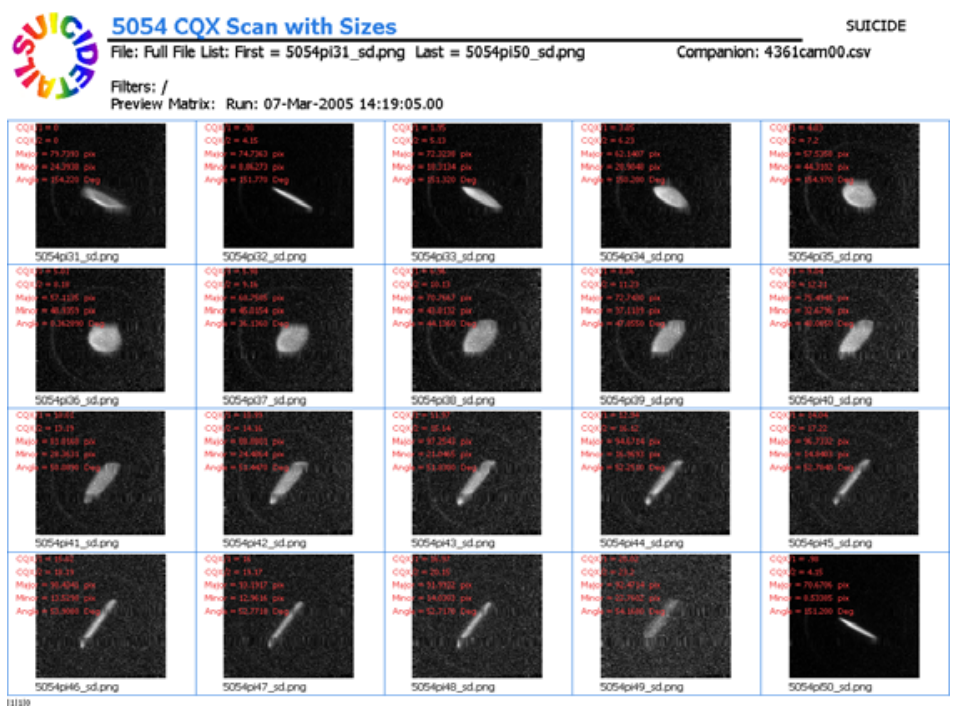

Figure 6.

A general observation on matching the codes to the experimental data was if we were only propagating ahead one or two magnets then the codes agreed fairly well with the observed data. However, when we tried to predict beam sizes (or shapes) after running through 4 or five magnets, then the quantitative measurements were not in as good agreement. For a full discussion on the matching of beam codes to experimental data refer to the paper by Frank Chambers et al in these proceedings (Parallel Measurement and Modeling of Transport in the DARHT II Beamline on ETA II).

During the entire course of these experiments, the LANL experimental team was in residence at Livermore and worked side by side with the LLNL experimental team. This included the set up and alignment of the DARHT II equipment in the ETA II accelerator hall and well as the initial debugging of magnet connections, control systems, and diagnostics. Once the experiment was up and running, the LANL team participated fully in the daily experiments. They were involved in the day to day planning and data collection and worked daily with their LLNL counterparts. By working with the equipment 
for five months they were able to learn its intricacies and they are now well prepared to run these systems when they are installed in the DARHT II accelerator hall.

\section{CONCLUSIONS}

We have completed a very successful experimental campaign using the DARHT II hardware at the ETA II facility. By scaling the ETA II current, we were able to use the DARTHT II hardware in its original configuration. The only alterations needed were the addition of one magnet and changing the diameter of one section of beam pipe. We have demonstrated shots on target using the Kicker and quadrupole transport system. We have used the LANL diagnostics and data acquisition system to control the Kicker and we have compared two transport codes (LAMBDA and FITS) to the experimental data and benchmarked these codes. Finally, we have completed a very successful technology transfer from LLNL to LANL and have reduced the time and effort that will be necessary at Los Alamos to bring these systems on line.

\section{GLOSSARY}

$\begin{array}{ll}\text { Beam Bug } & \text { Current and centroid monitor } \\ \text { CAMX } & \text { 4 Channel X-Ray pinhole camera } \\ \text { DARHT II } & \text { Dual Axis Radiographic HydroTest (2nd Axis) } \\ \text { ETA II } & \text { Experimental Test Accelerator (Version 2) } \\ \text { Kicker } & \text { Pulsed parallel plate beam deflector } \\ \text { Kicker Bias } & \\ \text { Dipole } & \text { Steering coil wrapped around the kicker } \\ \text { LANL } & \text { Los Alamos National Laboratory } \\ \text { LLNL } & \text { Lawrence Livermore National Laboratory }\end{array}$

